\title{
BMJ Open Randomised controlled trial protocol for the PROTECT-CS Study: PROTein to Enhance outComes of (pre)frail paTients undergoing Cardiac Surgery
}

\author{
Alexandra V Rose (D , , Todd Duhamel, ${ }^{1,2,3}$ Chris Hyde, ${ }^{1}$ Dave E Kent, ${ }^{1,4}$ \\ Jonathan Afilalo, ${ }^{5}$ Annette S H Schultz, ${ }^{6}$ Anna Chudyk (D) , 7 Dustin S Kehler, ${ }^{8}$ \\ Mudra Dave, ${ }^{1,4}$ Rakesh C Arora ${ }^{3,4,9}$
}

To cite: Rose AV, Duhamel T, Hyde C, et al. Randomised controlled trial protocol for the PROTECT-CS Study: PROTein to Enhance outComes of (pre) frail paTients undergoing Cardiac Surgery. BMJ Open 2021;11:e037240. doi:10.1136/ bmjopen-2020-037240

- Prepublication history is published online only. To view please visit the journal online (http://dx.doi.org/10.1136/ bmjopen-2020-037240).

Received 03 February 2020 Revised 03 December 2020 Accepted 22 December 2020

Check for updates

(c) Author(s) (or their employer(s)) 2021. Re-use permitted under CC BY-NC. No commercial re-use. See rights and permissions. Published by BMJ.

For numbered affiliations see end of article.

Correspondence to

Dr Rakesh C Arora;

rakeshcarora@gmail.com

\section{ABSTRACT}

Introduction In the past 20 years, the increasing burden of heart disease in an ageing population has resulted in cardiac surgery (CS) being offered to more frail and older patients with multiple comorbidities. Frailty and malnutrition are key geriatric syndromes that impact postoperative outcomes, including morbidity, mortality and prolonged hospital length of stay. Enhanced recovery protocols (ERPS), such as prehabilitation, have been associated with a reduction in complications after CS in vulnerable patients. The use of nutritional ERPs may enhance short-term and long-term recovery and mitigate frailty progression while improving patient-reported outcomes.

Methods and analysis This trial is a two-centre, doubleblinded, placebo, randomised controlled trial with blinded endpoint assessment and intention-to-treat analysis. Onehundred and fifty CS patients will be randomised to receive either a leucine-rich protein supplement or a placebo with no supplemented protein. Patients will consume their assigned supplement two times per day for approximately 2 weeks pre-procedure, during in-hospital postoperative recovery and for 8 weeks following discharge. The primary outcome will be the Short Physical Performance Battery score. Data collection will occur at four time points including baseline, in-hospital (pre-discharge), 2-month and 6-month time points post-surgery.

Ethics and dissemination The University of Manitoba Biomedical Research Ethics Board (20 March 2018) and the St Boniface Hospital Research Review Committee (28 June 2019) approved the trial protocol for the primary site in Winnipeg, Manitoba, Canada. The second site's (Montreal, Quebec) ethics has been submitted and pending approval from the Research Ethics and New Technology Development Committee for the Montreal Heart Institute (December 2020). Recruitment for the primary site started February 2020 and the second site will begin January 2021. Data gathered from the PROTein to Enhance outComes of (pre)frail paTients undergoing Cardiac Surgery Study will be published in peer-reviewed journals and presented at national and international conferences. Knowledge translation strategies will be created to share findings with stakeholders who are positioned to implement evidence-informed change.
Strengths and limitations of this study

- The PROTein to Enhance outComes of (pre)frail paTients undergoing Cardiac Surgery Study is a double-blinded, placebo-based, randomised controlled trial.

- Recruitment will take place in two high-volume Canadian cardiac surgery centres located in Winnipeg and Montreal.

- Involvement of patients and caregivers as research partners across the study's research cycle, through the formation of a patient advisory panel.

Relatively small sample size.

Potential study impact Malnutrition and frailty play a crucial role in post-CS recovery. Nutritional ERPs are increasingly being recognised as a clinically relevant aspect of perioperative care. As such, this trial is to determine if leucine-rich protein supplementation at key intervals can mitigate frailty progression and facilitate enhanced postoperative recovery.

Trial registration number ClinicalTrials.gov Registry (NCT04038294).

\section{INTRODUCTION}

By 2031, 25\% of Canadians will be older than 65 years. ${ }^{1}$ By extension, it is expected that greater numbers of frail (defined as a cumulative decline in multiple physiological systems resulting in a vulnerability to stressor events ${ }^{12}$ ) older adult individuals will be referred for a cardiac surgery procedure. ${ }^{34}$ While frailty is not necessarily synonymous with age, it is more prevalent among an older adult population. ${ }^{5}$ More specifically, in the last two decades, the increasing burden of heart disease has resulted in cardiac surgery being offered to older and more frail patients. ${ }^{3}$ Previous studies have demonstrated that patients with higher levels of frailty prevalence, ranging from $20 \%$ to $53 \%,{ }^{6-8}$ depending on the evaluation tool 
used, typically experience higher rates of postoperative morbidity, mortality and prolonged hospital length of stay with associated increased costs to the Canadian healthcare system. ${ }^{79-11}$ It has been previously identified that approximately $50 \%$ of older adults undergoing cardiac surgery are frail, resulting in a population who are at a higher risk for poor outcomes versus non-cardiac surgery patients. ${ }^{12} 13$ Furthermore, pre-frail (defined as a transitional physiological state between robustness and frailty, characterised by intermediate accumulation of physiological dysfunction, resulting in a minimal decline in physiological reserve and functional capacity, supported by a Clinical Frailty Scale (CFS) score equal to $3^{14-16}$ ) cardiac surgery patients are at an increased risk of adverse events such as increased duration of mechanical ventilation, morbidity and hospital stay, ${ }^{16}{ }^{17}$ as well as postoperative frailty progression that ultimately results in functional disability and worse healthrelated quality of life (HRQoL). ${ }^{18} 19$

Outcomes associated with frail cardiac surgery patients are impacted further by malnutrition (defined as an unintentional, nutritional intake imbalance (not necessarily a decreased intake)), which is evident in $20 \%$ of the patients presenting for cardiac surgery. ${ }^{20}{ }^{21}$ Chronic malnutrition which results from age-related physiological decline (ie, decreased mobility, sensory functions (olfaction and taste) and cognition), physiological challenges (ie, increased dependency and loneliness) and chronic illness (ie, cardiovascular, polypharmacy) ${ }^{22} 23$ in combination with perioperative starvation, which is the typical perioperative fasting, inflammatory and regenerative processes that induce an exponential nutritional demand resulting in an additional demand-supply imbalance (ie, a state of metabolic stress) can cause a further decline in health status and poor recovery to baseline functional capacity in already frail patients.

Malnutrition and frailty have shared risk factors with overlapping clinical presentation that exacerbate each syndrome. ${ }^{24-27}$ The previous standard preoperative protocol requires preoperative fasting for 6-8 hours (may extend to 8-12 hours). Further, a delay (mean of 2 days) in initiating postoperative nutritional support as well as an inadequacy of caloric intake (approximately $70 \%$ less than the recommended intake) has often been reported in cardiac surgery patients. ${ }^{28}{ }^{29}$ This perioperative underfeeding compound the pre-existing chronic malnutrition in frail older patients undergoing cardiac surgery. Malnutrition can manifest into a catastrophic fat-free muscle mass decline, that intensifies 'senile sarcopenia' (defined as the typical age-related involuntary muscle and organ degradation; typically, $1 \%-2 \%$ per year after 30 years).$^{30-32}$ This altogether results in an exponential loss of lean muscle mass (sarcopenia) and muscle function (dynapenia) with ageing. ${ }^{33-35}$ Importantly, even a minimal $(10 \%)$ loss of fat-free muscle mass was found to be associated with a progressive functional decline, increased mortality and increased utilisation of healthcare services (including premature institutionalisation) among community-dwelling older adults. ${ }^{35}$ Furthermore, the malnutrition-accelerated decline in lean muscle mass and muscle function are consistent features of frailty (coined as the biological substrate of frailty exacerbation $^{36}$ ). The frail-malnourished patients cannot mobilise enough amino acids to commensurate with the $400 \%$ increased demand required to synthesise proteins for wound healing, immune function and acute-phase reactants. ${ }^{37}$ To compound this issue, the metabolic and immune response to injury induces insulin resistance ${ }^{38}$ further aggravating a caloric deficient state. Ultimately, this perioperative demand-supply imbalance results in an extensive insult to a reserve-deficient physiological system (as in a typical frail patient), disproportionately declining the health status and limiting a patient's recovery to baseline functional capacity.

Providing protein-caloric supplementation preoperatively, postoperatively and post-hospital discharge is recommended by many consensus guidelines ${ }^{39}$ including: enhanced recovery protocols (ERPs), ${ }^{40}$ The European Society for Clinical Nutrition and Metabolism ${ }^{41}$ and the Canadian Nutritional Support Clinical practice guidelines. ${ }^{42}$ Nutritional supplementation can be viewed as a potentially modifiable factor that can alleviate the preoperative frailty-chronic malnutrition associated with vulnerability. Cardiac surgery patients, in addition to their cardiac disease and associated comorbidities, experience higher levels of frailty and malnutrition versus other non-cardiac surgery patients. ${ }^{43-45}$ There is a significant knowledge gap regarding the efficacy of nutritional prehabilitation in preserving functional capacity (ie, mitigating frailty progression) and promoting enhanced recovery, among (pre)frail older adults undergoing cardiac surgery.

Enhanced recovery protocols, such as prehabilitation, have been associated with a reduction in overall complications and length of stay of up to $50 \%$ in vulnerable patients when compared with conventional perioperative patients in non-cardiac surgery populations. ${ }^{46-48}$ Early feasibility studies have shown promise with ERPs in cardiac surgery as well, however, require additional rigorous inquiry. Specifically, the impact of a nutritional supplementation ERP on mitigating long-term frailty progression and improving patient-reported outcomes (patient valued survivorship and recovery) in an increasingly ageing cardiac surgery patient population has been largely understudied. In Canada, when a patient requires elective cardiac surgery, they are placed on a 'waiting list' for as long as $3-4$ months. At present, there is no formal process for engaging these patients to enhance their health during this waiting period, which represents a significant opportunity to optimise a (pre)frail older adult patient's clinical condition prior to surgery. Additionally, the consideration of longer term (not just in-hospital) HRQoL is vitally important to the patient-caregiver unit. As a result, it is becoming critically important for the healthcare system to develop strategies to improve clinical outcomes in this high-risk patient population.

The PROTein to Enhance outComes of (pre)frail paTients undergoing Cardiac Surgery (the PROTECT-CS 
Study) was developed as a practical and sustainable, new perioperative care pathway, informed by patientcaregiver values, to enhance short-term and long-term recovery of vulnerable cardiac surgery patients.

The primary objectives of the trial are:

1. To determine if a leucine-rich protein supplementation, consumed two times per day for a minimum of 7 days pre-procedure for elective patients (minimum of 2 days for non-emergent inpatients), two times per day during in-hospital postoperative recovery and two times per day for 8 weeks after the patient is discharged home can reduce perioperative functional decline among (pre)frail older cardiac surgery patients.

2. To determine if a perioperative leucine-rich protein supplementation will enhance short-term and longer term patient-reported outcomes.

We hypothesise that the preoperative and postoperative supplementation of leucine-rich-formulated beverages to a patient's habitual diet will support the physiological processes to counter the impact of cardiac surgery-related stressors to reduce the postoperative functional decline. We also hypothesise that nutrition supplementation using a leucine-rich protein supplementation will facilitate postoperative recovery and improve self-reported HRQoL in (pre)frail cardiac surgery patients.

\section{POTENTIAL IMPACT OF STUDY}

To date, no high-quality study has prospectively examined the impact of perioperative nutrition supplementation in the higher risk, (pre)frail older adult undergoing cardiac surgery. The impact of the PROTECT-CS Study may provide a much-needed framework to direct future surgical practice and to inform ERP guidelines in the cardiac surgery patient. The PROTECT-CS Study is highly relevant and aims to improve the patient-centred and patient-driven perioperative approach for the optimisation of the (pre)frail older adult undergoing cardiac surgery. We endeavour to ensure patients do not just 'survive but thrive' after their heart surgery.

\section{METHODS AND ANALYSIS}

The PROTECT-CS Study is a patient/researcher collaborative study, involving two Canadian centres. The study is a double-blinded, placebo randomised controlled trial (RCT) with blinded endpoint assessment and intentionto-treat analysis. Randomisation of the placebo and supplement is stratified by site and sex. Additionally, a third party will be randomising the patients for this study to ensure double blinding. At present, there is no formal process for engaging patients undergoing cardiac surgery to enhance their health during their waiting period. There is a significant knowledge gap regarding the efficacy of nutritional supplementation in preserving functional capacity and promoting enhanced recovery, among (pre) frail older adults undergoing cardiac surgery. Improved perioperative processes that address frailty and proper nutrition are of significant value to the patient-caregiver unit. This study is funded by the Heart and Stroke Foundation of Canada. We have used the Standard Protocol Items: Recommendations for Interventions Trials guidelines, the Sex and Gender Equity in Research guidelines and the Consolidated Standards of Reporting Trials guidelines in reporting this clinical trial. A description of this clinical trial is available on http://ClinicalTrials.gov.

\section{PATIENT AND PUBLIC INVOLVEMENT}

Patient engagement in research involves meaningful and active collaborations between patients and researchers throughout the different phases of a research project, including planning, study design, data collection, data analysis and knowledge translation (KT) ${ }^{49}$ We have recruited 10 patients and caregivers to collaborate as research partners on this study through membership on an advisory panel. Panel members were recruited from a database of previous cardiac surgery patients who agreed to be contacted for future research. We selectively chose panel members to reflect key demographic characteristics of our study's target population. We anticipate that we will collaborate with the advisory panel across the study's entire research cycle-from study planning through to the end of study KT (3 years)-predominantly through face-to-face meetings. Figure 1 outlines the different areas that the advisory panel may contribute to the study. These include providing input on study implementation refinement and procedures (eg, recruitment methods, approaches to maximise adherence to supplement-intake protocols, use of accessible language in recruitment and study materials, and usability of technology), relevance of outcomes to patient concerns, data analysis (eg, interpretation and contextualisation of findings) and KT (eg, alternate dissemination methods, use of accessible language). ${ }^{50}$

\section{STUDY SETTING}

The PROTECT-CS trial will be conducted and recruited in two sites across Canada: (1) Winnipeg, Manitoba, St Boniface Hospital; (2) Montreal, Quebec, Jewish General Hospital.

\section{RECRUITMENT}

This study will recruit 150 patients over a 24-month recruitment period, starting January 2020, between both sites (ie, four per month for the primary site and two per month for the second site). We expect a total recruitment rate of approximately $20 \%-25 \%$ of potentially eligible patients based on previous research ${ }^{30}$ (figure 2).

\section{ELIGIBILITY CRITERIA \\ Screening}

Initial screening for the study will include the CFS, the Short Performance Physical Battery (SPPB) and the Short 


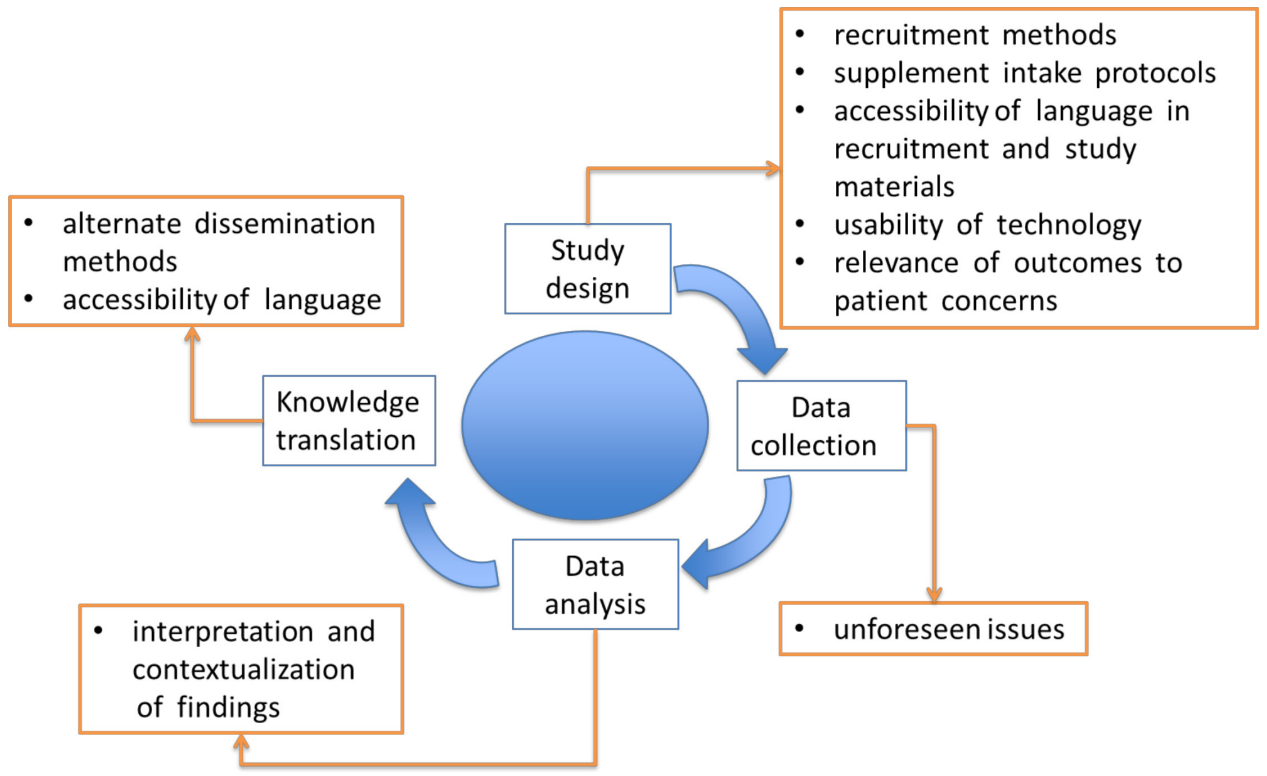

Figure 1 Anticipated contributions of the patient advisory panel.

Form-36 Physical Function (SF-36-PF) survey to measure frailty status in potential study patients. We have defined a CFS of greater than or equal to 3 (classified as 'managing well'), but not 7 (severely frail) or higher as an initial indicator of frailty for the purposes of our study's inclusion criteria. An SPPB score of less than or equal to 9 or a score of $\leq 60$ on the SF-36-PF questionnaire for suitable participation. If the screening criteria are met, the research team will obtain signed consent, administer all baseline assessments and randomly allocate each patient to the appropriate research group (ie, protein or placebo). Patients who do not meet the frailty inclusion criteria are excluded based on specific criteria or refuse to be randomised into the main study will be given the opportunity to participate in the registry component of the study (figure 3 ).

\section{OUTCOMES AND INSTRUMENTATION}

The primary outcome is a change in the SPPB score and the SF-36-PF score at 2 (short) and 6 (longer term) months compared with baseline. Due to the global pandemic (COVID-19), research initiatives that collected data through in-person meetings were suspended on two occasions (March 2020-August 2020, and November 2020—to be discussed (TBD)). As a result, we developed an alternative primary outcome (ie, SF-36-PF) to implement for the trial in order to accommodate the institutional directive to transition research to use remote/virtual data collection strategies. The SF-36-PF is an optimal outcome measure appropriate for our study cohort, as it is correlated with the SPPB $(r=0.5-0.6)$ and is a surrogate measure of physical function. ${ }^{51}$ Secondary outcomes are adherence to supplementation, fat-free muscle mass (measured by a portable bioimpedance device); HRQoL measured by the EQ-5D-3L, EQ-Visual Analogue Scale (EQ-VAS) ${ }^{52}$ and the Older American Resource Scale (OARS): Activities of Daily Living (ADL) and Instrumental ADL (IADL) questionnaire ${ }^{53}$; mood as measured by the Patient Health Questionnaire-9 (PHQ-9 $)^{54}$; current nutrition as measured by the Mini Nutrition Assessment (MNA) tool ${ }^{55}$; anxiety as measured by the Cardiac Anxiety Questionnaire $(\mathrm{CAQ})^{56}$; self-reported physical activity, exhaustion and nutrition as assessed by the Modified Fried Questionnaire ${ }^{26}$; physical activity accumulation as measured by actical accelerometers, aerobic fitness as measured by the 6 Min Walk Test $(6 \mathrm{MWT})^{57}$; and a composite safety endpoint of all-cause mortality, injurious fall, acute kidney injury or readmission

\section{Estimated potentially enrollable patients from both sites $n=\sim 804$ patients over 24 months.}

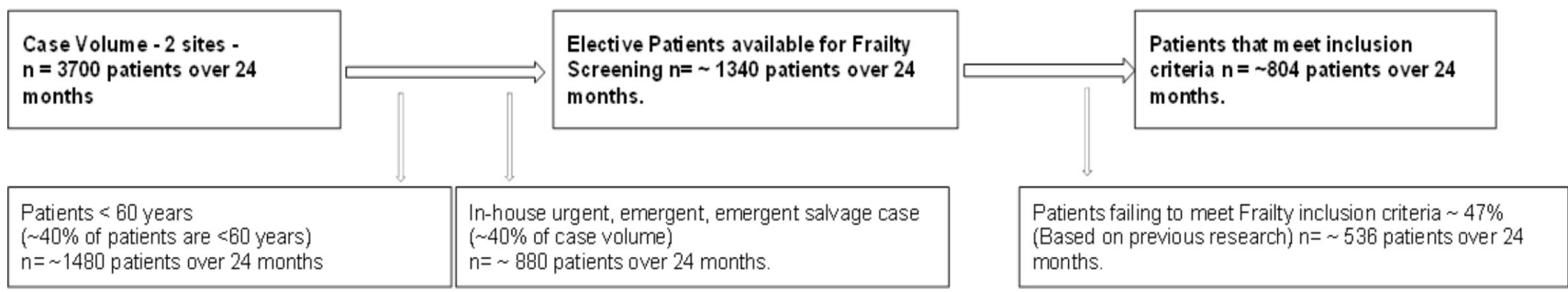

Figure 2 Potential recruitment numbers. 
Box 1 Inclusion and exclusion criteria for the PROTECTCS trial

\section{Inclusion criteria}

- Patients aged 60 years or older, undergoing elective or nonemergent isolated CABG, aortic valve repair or replacement for moderate aortic stenosis or severe regurgitation, mitral valve repair or replacement for moderate mitral stenosis or severe regurgitation, or combined CABG/valve procedures.

- Patient with a Clinical Frailty Scale (CFS) from 3 (managing well) to 6 (moderately frail). ${ }^{1467}$

- Patients with an estimated wait time of approximately 7 days or longer for elective surgery or 2 days or longer for non-emergent surgery.

\section{Exclusion criteria}

- Decompensated or non-ambulatory class IV symptoms of angina, dyspnoea and claudication.

- Patients with a CFS of 7 or greater (severely frail to terminally ill); this will exclude less than $1 \%$ of the population on the elective cardiac surgery waitlist. ${ }^{52}$

- Creatinine clearance $<30 \mathrm{~mL} / \mathrm{min} / 1.83 \mathrm{~m}^{2}$

- Cirrhosis (Child-Pugh class B or greater).

- Allergy to milk proteins or other ingredients in the supplement.

- Inability to safely ingest beverages by mouth.

- Mild-to-severe cognitive impairment (ie, Montreal Cognitive Assessment <16). ${ }^{67}$

- An inability to speak/read in English or French.

- Emergent patients or non-emergent patients going for surgery within 48 hours of admission to hospital.

CABG, coronary artery bypass grafting; PROTECT-CS, PROTein to Enhance outComes of (pre)frail paTients undergoing Cardiac Surgery.

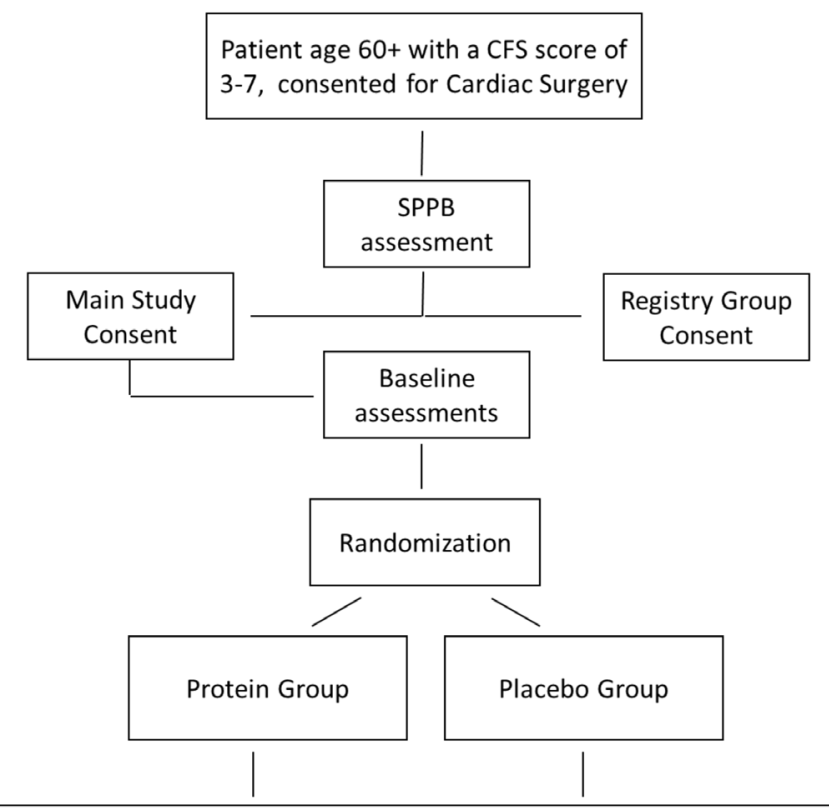

4 Research visits - 1: Baseline, 2: post-surgery discharge from hospital, 3: 2-month post surgery and 4: 6-month post surgery

Figure 3 PROTECT-CS screening and allocation plan. CFS, Clinical Frailty Scale; PROTECT-CS, PROTein to Enhance outComes of (pre)frail paTients undergoing Cardiac Surgery; SPPB, Short Performance Physical Battery. for related events at 2 months provided by medical records. The primary and secondary outcomes will be assessed at baseline prior to cardiac surgery procedure and reassessed at 2 and 6 months post-hospital discharge. Additionally, the SPPB, SF-36-PF, fat-free muscle mass (assessed by bioelectrical impedance analysis (BIA)) and the MNA, and an assessment of nausea and vomiting symptoms will be reassessed while in-hospital prior to discharge if appropriate. It should be noted, the portable BIA device uses segmental multifrequency technology to measure muscle mass with an accuracy comparable with dual X-ray absorptiometry ${ }^{27}$; and while the accuracy is inferior to that of CT and MRI, it is endorsed by consensus guidelines as a 'good portable alternative that is inexpensive, easy to use, and readily reproducible'.

\section{BLINDING}

Patients will be blinded to the treatment group allocation, as well as the research staff who will assess the fatfree muscle mass, the SPPB and other endpoints at each time point to avoid ascertainment bias.

\section{STUDY INTERVENTION}

\section{Study participants}

All patients consenting to the study will be randomly allocated to either a protein or placebo group and will undergo the standard preoperative evaluation for suitability and planning for their cardiac surgery procedures. Patients will then be placed on the surgical waitlist. At the time of their cardiac surgery, both the placebo and protein group participants will ingest a carbohydrate beverage, PREcovery, (ie, $50 \mathrm{~g}$ of complex carbohydrates; aka CHO loading) 2-3hours before surgery. The carbohydrate beverage consumed before surgery reduces insulin resistance and tissue glycosylation, improves postoperative glucose control, reduces nausea and vomiting, and also enhances the return of gastrointestinal function post-surgery. ${ }^{58} 59$

\section{Protein group}

Patients randomised to the protein group will receive a leucine-rich protein supplement derived and provided by Enhanced Medical Nutrition (https://emnhealth. $\mathrm{com} /$ ). The product contains $25 \mathrm{~g}$ protein and $3 \mathrm{~g}$ leucine per serving (total caloric value: $160 \mathrm{Kcal}$ ) to be reconstituted and consumed two times per day for a minimum of 2 weeks pre-procedure, two times per day during postoperative in-hospital recovery ( $\sim 5-10$ days) and two times per day for 8 weeks after the patient is discharged home. The supplement will be consumed approximately 1-2 hours after the morning meal and also 1-2 hours after lunch or before bedtime so as to supplement rather than replace meals. The protein supplement (ISOlution) is formulated to minimise appetite suppression. A research assistant, who is blinded to group allocation, will provide the patient's supply of supplements at the time of study consent (supplementation prior to cardiac surgery) and after discharge from the hospital (supplementation after 
cardiac surgery). In-hospital supplementation will be provided to the patient directly from research staff on a daily basis until discharged home. Phone calls will also be made to participants once per week to ensure compliance. Patients will be encouraged to perform light-tomoderate-intensity aerobic activity up to $30 \mathrm{~min}, 5$ days per week as tolerated based on the 2016 edition of 'Living well with heart disease', published by the Heart and Stroke Foundation of Canada. ${ }^{60}$ This brochure outlines practical tips to safely begin a walking programme and gradually increase walking time to meet the goal of 150 min per week.

\section{Placebo group}

Enrolled patients allocated to the placebo group will receive the same supplementation schedule followed by the protein group, as well as compliance verification; however, they will receive a placebo product with no supplemented protein (no additional nutritional benefit). This placebo product derived and provided by the Enhanced Medical Nutrition (https://emnhealth. $\mathrm{com} /$ ) will look exactly like the protein-rich supplement. A research assistant, who is blinded to group allocation, will provide the patient's supply of supplements at the time of study consent (supplementation prior to cardiac surgery) and after discharge from the hospital (supplementation after cardiac surgery). In-hospital supplementation will be provided to the patient directly from research staff on a daily basis until discharged home. Phone calls will also be made to participants once per week to ensure compliance. Additionally, patients will be encouraged to perform light-to-moderate-intensity aerobic activity up to $30 \mathrm{~min}, 5$ days per week as tolerated based on the 2016 edition of 'Living well with heart disease', published by the Heart and Stroke Foundation of Canada. ${ }^{60}$ This brochure outlines practical tips to safely begin a walking programme and gradually increase walking time to meet the goal of $150 \mathrm{~min}$ per week.

\section{Registry group}

Patients undergoing cardiac surgery who want to participate in the research but refuse to be randomised into the protein group or the placebo group, or those who want to participate in the research but are excluded based on specific study criteria, will be invited to participate in a registry group that will be followed for the purpose of documenting the effects of standard care. Registry participants will not be required to consume study supplements or CHO prior to surgery. Participants will be asked to complete basic demographic and quality of life questionnaires during their consent process and at 2 and 6 months after their cardiac surgery. Additionally, prior to their discharge from the hospital after their cardiac surgery, they will be asked about their postoperative experience specifically related to nausea, vomiting and nutrition while in hospital.

\section{DATA COLLECTION AND MANAGEMENT}

Participants will meet with the research staff for data collection at four separate points. This will include after consenting to their surgical procedure (baseline presurgery), during the in-hospital stay (postoperatively prior to discharge), and at the 2-month and 6-month time point post-surgery. The SPPB and SF-36-PF will be assessed to determine frailty status change at discharge, 2-month and 6 -month post-surgery. The 6MWT, hand grip assessment and Fried Questionnaires will also be used to assess frailty in study participants at the above time points. Additionally, nutrition will be assessed by the MNA questionnaire at baseline, in-hospital (postoperatively prior to discharge), at 2-month and 6-month post-surgery. During the in-hospital research visit, all participants' nausea and vomiting symptoms will be assessed by research staff. Objective measurements of fat-free muscle mass will be obtained by using a BIA device which will be assessed at baseline, in-hospital (postoperatively prior to discharge), and during 2-month and 6-month post-surgery follow-up visits. Physical activity, sedentary behaviour and compliance to physical activity recommendations will be assessed by an accelerometer that participants will wear for a period of 7 days at baseline and at 2-month and 6-month post-surgery follow-up appointments. As COVID-19-related restrictions continue to persist, the potential of in-person visits is diminished based on institutional protocols. Therefore, assessment of physical parameters may not be possible for each research appointment. However, survey administration will continue for all research time points.

The EQ-5D-3L, the EQ-VAS, and the OARS ADL and IADL will assess HRQoL at baseline, 2-month and 6-month post-surgery time points. The PHQ-9 and the CAQ will be administered at baseline, 2-month and 6-month postsurgery time points.

Sex and gender data will be collected at baseline and analysed to identify relationships as they occur within this cohort of cardiac surgery patients. We will capture this information through questionnaires as part of the survey package presented to research participants. Specifically, participants will be asked: (1) what is your assigned sex? (forced choice: male, female or intersex) and (2) what is your gender identity? (man, woman or please specify (openended response)). It has been previously published that gender and sex differences are a consideration regarding risk for and outcomes of cardiovascular disease. ${ }^{61}$

\section{Sample size}

Based on a previously observed distribution of SPPB scores in our elective cardiac surgery population, we expect this effect size will be approximately equivalent to either a 1-point change in the SPPB score or a 10-point change in SF-36-PF score. This magnitude of change in SPPB score is associated with a'substantial' improvement in mobility and quality of life, ${ }^{27}$ and survival. ${ }^{62}$ Furthermore, the scale of change for the SF-36-PF is sensitive to detect even a small and clinically meaningful change in physical functioning. ${ }^{63}$ Due to the global pandemic 
(COVID-19), research initiatives that collected data through in-person meetings were suspended on two occasions (March 2020-August 2020, and November 2020TBD). As a result, we developed an alternative primary outcome (ie, SF-36-PF) to implement for the trial in order to accommodate the institutional directive to transition research to use remote/virtual data collection strategies. The SF-36-PF is an optimal outcome measure appropriate for our study cohort, as it is correlated with the SPPB $(\mathrm{r}=0.5-0.6)$ and is a surrogate measure of physical function. ${ }^{51}$ A sample size of 150 patients (75 in each group) will allow for an overall drop-out rate of $15 \%(n=22)$ while maintaining a moderate Cohen's effect size (0.5) to detect the difference in change experienced between the two experimental groups at each follow-up time point with a two-tailed alpha of 0.05 and a power of $80 \%$ for the continuous primary outcomes. G*Power V.3.1 was used to calculate sample size for this study.

\section{Statistical methods}

The primary analysis for this project will be intentionto-treat. A secondary a priori per-protocol analysis will be performed to assess the efficacy of the protein-rich nutritional supplement on the primary and secondary outcomes. Continuous outcome variables measured at multiple time points will be analysed using a repeated measure analysis of covariance (ANCOVA) considering site and sex as fixed effects. Additional covariates such as age, comorbidities and baseline cardiac function may be considered in the ANCOVA analysis if other imbalances remain following randomisation. Post hoc analysis that applies a Bonferroni correction to control the familywise error rate will be performed to identify how the primary and secondary outcomes change at each time point. Categorical outcome variables will be compared using a $\mathrm{X}^{2}$ test or Fisher's exact test where appropriate. A sex-based and gender-based analysis will be performed by either stratifying the analysis or by including sex and gender as independent factors in the main analysis. Standardised mean differences will be calculated for several important perioperative characteristics to assess the covariate balance between study groups. Inverse probability of treatment weighting or multivariable regression techniques may also be considered if the ANCOVA assumptions are violated. ${ }^{64}$ The study biostatistician, who is experienced in RCT methodology, will oversee all statistical analyses.

\section{Data monitoring}

A research coordinator will work out of the I H Asper Clinical Research Institute at the St Boniface Hospital/Research Centre, where the principal investigator and co-investigator are primarily appointed. Data management services, statistical and methodological support are to be housed within the Cardiac Sciences Program at the St Boniface Hospital. The research coordinator will assume responsibility for the data handling. The REDCap platform, housed on a virtual private network at the University of Manitoba, has been used for data collection to ensure safe storage of electronic data for both study sites.

\section{ETHICS AND DISSEMINATION \\ Informed consent}

Patients will first consent to their surgical procedure before being considered for study participation. The patient will be approached and informed about the trial by the research assistant and provided with a copy of the patient information and consent form. Patients will be given adequate amount of time to consider their participation in the trial and will be given an opportunity to ask questions if needed. If the patient decides to participate in the study, they will be asked to provide written consent. All participants are free to withdraw from the study at any time, without any prejudice to future medical treatment.

\section{Trial monitoring and safety}

The study collaborators are responsible for ensuring proposed milestones and deadlines are met. They are also responsible for study design, management, ethical conduct, analysis and dissemination of results. Safety data, including new hospitalisation, worsening heart symptoms and other adverse events will be captured and reportable to the study Data and Safety Monitoring Board (DSMB) as they occur. The DSMB is an independent group of experts that advises study investigators. They are responsible for a periodical evaluation of the study data (ie, every 6 months) for participant safety and study conduct, in addition to making recommendations concerning the modification and/or termination of the trial.

\section{Dissemination}

Results will be distributed to an interdisciplinary team of cardiologists, cardiac surgeons, geriatricians, nurses, dieticians, rehabilitation providers, policymakers and patients by way of printed and electronic educational materials as well as oral presentation at regional meetings. Furthermore, we have formulated an integrated KT (iKT) team with a dedicated patient panel. Our iKT strategy will seek to adapt the intervention to local resources and expertise by including clinicians, dieticians and patient panels to develop a clinical pathway for the institution of treatment strategies with a mean of evaluation of effectiveness using the Plan-DoStudy-Act cycle ${ }^{65}$ (a framework for developing, testing and implementing changes leading to improvement). We will validate our results in a larger multicentre trial leveraging ERAS Cardiac Surgery Society and CANCARE Society participating sites. In addition, we will organise focus group sessions with the patient-caregiver unit and our industry partner to seek their input to further refine and improve the leucine-rich protein-caloric supplement. In doing so, patients can transform the research process from one directed by investigators to one driven and informed by the needs of patients and their caregivers. Patient engagement is recognised as a necessary approach to the building of a 
sustainable and equitable healthcare system and to improve health outcomes. ${ }^{66}$

\section{Author affiliations}

${ }^{1}$ Faculty of Kinesiology and Recreation Management, University of Manitoba, Winnipeg, Manitoba, Canada

${ }^{2}$ Health, Leisure and Human Performance Research Institute, University of Manitoba, Winnipeg, Manitoba, Canada

${ }^{3}$ Institute of Cardiovascular Sciences, St. Boniface General Hospital Albrechtsen Research Centre, Winnipeg, Manitoba, Canada

${ }^{4}$ Cardiac Science Manitoba, St. Boniface Hospital, Winnipeg, Manitoba, Canada

${ }^{5}$ Department of Medicine, McGill University, Montreal, Quebec, Canada

${ }^{6}$ College of Nursing, University of Manitoba, Winnipeg, Manitoba, Canada

${ }^{7}$ Department of Family Medicine, University of Manitoba, Winnipeg, Manitoba,

Canada

${ }^{8}$ Department of Physiotherapy, Dalhousie University, Halifax, Nova Scotia, Canada

${ }^{9}$ Department of Surgery, University of Manitoba, Winnipeg, Manitoba, Canada

\section{Twitter Rakesh C Arora @TheRakeshArora}

Acknowledgements This research study acknowledges the support of the cardiac surgery healthcare staff and research offices at the St Boniface Hospital. We also wish to acknowledge and thank our patient collaborators for their contributions to the design and direction of this study. By using patient collaboration, we were able to design a study supported by patients for patients. The patient collaborative efforts were invaluable to our research group.

Contributors RCA and TD are co-senior authors. AVR and DEK were involved in the manuscript preparations. CH, DEK, JA, ASHS, AC, DSK and MD were involved in the conception of the study design, AVR, CH, DEK, JA, ASHS, AC, DSK and MD were involved in the manuscript revisions.

Funding This work was supported by The Heart and Stoke Foundation of Canada, grant number G-19-0024269. RCA has received an unrestricted educational grant from Pfizer Canada and received honoraria from Abbott Nutrition for work unrelated to this manuscript.

\section{Competing interests None declared.}

Patient and public involvement Patients and/or the public were involved in the design, or conduct, or reporting, or dissemination plans of this research. Refer to the Methods section for further details.

Patient consent for publication Not required.

Provenance and peer review Not commissioned; externally peer reviewed.

Open access This is an open access article distributed in accordance with the Creative Commons Attribution Non Commercial (CC BY-NC 4.0) license, which permits others to distribute, remix, adapt, build upon this work non-commercially, and license their derivative works on different terms, provided the original work is properly cited, appropriate credit is given, any changes made indicated, and the use is non-commercial. See: http://creativecommons.org/licenses/by-nc/4.0/.

\section{ORCID iDs}

Alexandra V Rose http://orcid.org/0000-0003-4600-2848

Anna Chudyk http://orcid.org/0000-0001-8476-2093

\section{REFERENCES}

1 Statistics C. National seniors Day.By the numbers, 2014. Available: http://www.statcan.gc.ca/eng/dai/smr08/2014/smr08_191_2014 [Accessed 6 Dec 2017].

2 Fried LP, Hadley EC, Walston JD, et al. From bedside to bench: research agenda for frailty. Sci Aging Knowledge Environ 2005;2005:pe24.

3 Ferguson TB, Hammill BG, Peterson ED, et al. A decade of change-risk profiles and outcomes for isolated coronary artery bypass grafting procedures, 1990-1999: a report from the STS Nationa Database Committee and the Duke Clinical Research Institute. Society of Thoracic Surgeons. Ann Thorac Surg 2002;73:480-9.

4 Abramov D, Tamariz MG, Fremes SE, et al. Trends in coronary artery bypass surgery results: a recent, 9-year study. Ann Thorac Surg 2000;70:84-90

5 Afilalo J, Alexander KP, Mack MJ, et al. Frailty assessment in the cardiovascular care of older adults. J Am Coll Cardiol 2014;63:747-62.
6 Afilalo J, Mottillo S, Eisenberg MJ, et al. Addition of frailty and disability to cardiac surgery risk scores identifies elderly patients at high risk of mortality or major morbidity. Circ Cardiovasc Qual Outcomes 2012;5:222-8.

7 Sündermann S, Dademasch A, Rastan A, et al. One-Year follow-up of patients undergoing elective cardiac surgery assessed with the comprehensive assessment of frailty test and its simplified form. Interact Cardiovasc Thorac Surg 2011;13:119-23.

8 Lytwyn J, Stammers AN, Kehler DS, et al. The impact of frailty on functional survival in patients 1 year after cardiac surgery. $J$ Thorac Cardiovasc Surg 2017;154:1990-9.

9 Green P, Woglom AE, Genereux P, et al. The impact of frailty status on survival after transcatheter aortic valve replacement in older adults with severe aortic stenosis: a single-center experience. JACC Cardiovasc Interv 2012;5:974-81.

10 Lee DH, Buth KJ, Martin B-J, et al. Frail patients are at increased risk for mortality and prolonged institutional care after cardiac surgery. Circulation 2010;121:973-8.

11 Singh M, Rihal CS, Lennon RJ, et al. Influence of frailty and health status on outcomes in patients with coronary disease undergoing percutaneous revascularization. Circ Cardiovasc Qual Outcomes 2011:4:496-502.

12 Hewitt J, Long S, Carter B, et al. The prevalence of frailty and its association with clinical outcomes in general surgery: a systematic review and meta-analysis. Age Ageing 2018;47:793-800.

13 Robinson TN, Wallace JI, Wu DS, et al. Accumulated frailty characteristics predict postoperative discharge institutionalization in the geriatric patient. J Am Coll Surg 2011;213:37-42.

14 Rockwood K, Song X, MacKnight C, et al. A global clinical measure of fitness and frailty in elderly people. CMAJ 2005;173:489-95.

15 Sergi G, Veronese N, Fontana L, et al. Pre-frailty and risk of cardiovascular disease in elderly men and women: the Pro.V.A. study. J Am Coll Cardiol 2015;65:976-83.

16 Fontana L, Addante F, Copetti M, et al. Identification of a metabolic signature for multidimensional impairment and mortality risk in hospitalized older patients. Aging Cell 2013;12:459-66.

17 Rodrigues MK, Marques A, Lobo DML, et al. Pre-Frailty increases the risk of adverse events in older patients undergoing cardiovascular surgery. Arq Bras Cardiol 2017;109:299-306.

18 Walston J, Hadley EC, Ferrucci L, et al. Research agenda for frailty in older adults: toward a better understanding of physiology and etiology: summary from the American geriatrics Society/National Institute on aging research conference on frailty in older adults. $J$ Am Geriatr Soc 2006;54:991-1001.

19 Arya S, Long CA, Brahmbhatt R, et al. Preoperative frailty increases risk of Nonhome discharge after elective vascular surgery in homedwelling patients. Ann Vasc Surg 2016;35:19-29.

20 Pathirana AK, Lokunarangoda N, Ranathunga I, et al. Prevalence of hospital malnutrition among cardiac patients: results from six nutrition screening tools. Springerplus 2014;3:412-7.

21 McWhirter JP, Pennington CR. Incidence and recognition of malnutrition in hospital. BMJ 1994;308:945-8.

22 Heuberger RA, Caudell K. Polypharmacy and nutritional status in older adults: a cross-sectional study. Drugs Aging 2011;28:315-23.

23 Ortolani E. Nutritional status and drug therapy in older adults. $J$ Gerontol Geriatric Res 2013;02:2-5.

24 Laur CV, McNicholl T, Valaitis R, et al. Malnutrition or frailty? overlap and evidence gaps in the diagnosis and treatment of frailty and malnutrition. Appl Physiol Nutr Metab 2017;42:449-58.

25 Vellas B, Cesari M, Li J. The white book on frailty. 1, 2016.

26 Fried LP, Tangen CM, Walston J, et al. Frailty in older adults: evidence for a phenotype. J Gerontol A Biol Sci Med Sci 2001;56:M146-57.

27 Jeejeebhoy KN, Malnutrition JKN. Malnutrition, fatigue, frailty, vulnerability, sarcopenia and cachexia: overlap of clinical features. Curr Opin Clin Nutr Metab Care 2012;15:213-9.

28 Rahman A, Agarwala R, Martin C, et al. Nutrition therapy in critically ill patients following cardiac surgery: defining and improving practice. JPEN J Parenter Enteral Nutr 2017;41:1188-94.

29 Drover JW, Cahill NE, Kutsogiannis J, et al. Nutrition therapy for the critically ill surgical patient: we need to do better! JPEN J Parenter Enteral Nutr 2010;34:644-52.

30 Morley JE. Anorexia, sarcopenia, and aging. Nutrition 2001;17:660-3.

31 Ferrucci L, de Cabo R, Knuth ND, et al. Of Greek heroes, wiggling worms, mighty mice, and old body builders. J Gerontol A Biol Sci Med Sci 2012;67:13-16.

32 Goodpaster BH, Park SW, Harris TB, et al. The loss of skeletal muscle strength, mass, and quality in older adults: the health, aging and body composition study. J Gerontol A Biol Sci Med Sci 2006:61:1059-64. 
33 Cederholm T, Bosaeus I, Barazzoni R, et al. Diagnostic criteria for malnutrition - An ESPEN Consensus Statement. Clin Nutr 2015;34:335-40.

34 Genton L, Karsegard VL, Chevalley T, et al. Body composition changes over 9 years in healthy elderly subjects and impact of physical activity. Clin Nutr 2011;30:436-42.

35 Broadwin J, Goodman-Gruen D, Slymen D. Ability of fat and fat-free mass percentages to predict functional disability in older men and women. J Am Geriatr Soc 2001;49:1641-5.

36 Landi F, Calvani R, Cesari M, et al. Sarcopenia as the biological substrate of physical frailty. Clin Geriatr Med 2015;31:367-74.

37 Wolfe RR. Optimal nutrition, exercise, and hormonal therapy promote muscle anabolism in the elderly. J Am Coll Surg 2006;202:176-80.

38 Noblett SE, Watson DS, Huong $\mathrm{H}$, et al. Pre-Operative oral carbohydrate loading in colorectal surgery: a randomized controlled trial. Colorectal Dis 2006;8:563-9.

39 Stoppe C, Goetzenich A, Whitman G, et al. Role of nutrition support in adult cardiac surgery: a consensus statement from an international multidisciplinary expert group on nutrition in cardiac surgery. Crit Care 2017;21:131.

40 Carmichael JC, Keller DS, Baldini G, et al. Clinical practice guidelines for enhanced recovery after colon and rectal surgery from the American Society of colon and rectal surgeons and society of American gastrointestinal and endoscopic surgeons. Dis Colon Rectum 2017;60:761-84.

41 Weimann A, Braga M, Carli F, et al. ESPEN guideline: clinical nutrition in surgery. Clin Nutr 2017;36:623-50.

42 Heyland DK, Dhaliwal R, Drover JW, et al. Canadian clinical practice guidelines for nutrition support in mechanically ventilated, critically ill adult patients. JPEN J Parenter Enteral Nutr 2003;27:355-73.

43 Afilalo J. Frailty in patients with cardiovascular disease: why, when, and how to measure. Curr Cardiovasc Risk Rep 2011;5:467-72.

44 Newman AB, Gottdiener JS, Mcburnie MA, et al. Associations of subclinical cardiovascular disease with frailty. J Gerontol A Biol Sci Med Sci 2001;56:M158-66.

45 Klein BEK, Klein R, Knudtson MD, et al. Frailty, morbidity and survival. Arch Gerontol Geriatr 2005;41:141-9.

46 Spanjersberg WR, Reurings J, Keus F, et al. Fast track surgery versus conventional recovery strategies for colorectal surgery. Cochrane Database Syst Rev 2011:CD007635.

47 Thiele RH, Rea KM, Turrentine FE, et al. Standardization of care: impact of an enhanced recovery protocol on length of stay, complications, and direct costs after colorectal surgery. J Am Coll Surg 2015;220:430-43.

48 Stone AB, Grant MC, Pio Roda C, et al. Implementation costs of an enhanced recovery after surgery program in the United States: a financial model and sensitivity analysis based on experiences at a quaternary academic medical center. J Am Coll Surg 2016;222:219-25.

49 Canadian Institute for Health Research. Strategy for Patient-Oriented Research - Patient Engagement Framework 2014.
50 Shippee ND, Domecq Garces JP, Prutsky Lopez GJ, et al. Patient and service user engagement in research: a systematic review and synthesized framework. Health Expect 2015;18:1151-66.

51 Berry MJ, Love NJ, Files DC, et al. The relationship between self-report and performance-based measures of physical function following an ICU stay. J Crit Care 2019;51:19-23.

52 EuroQol Group. EuroQol--a new facility for the measurement of health-related quality of life. Health Policy 1990;16:199-208.

53 Fillenbaum GG, Smyer MA. The development, validity, and reliability of the OARS multidimensional functional assessment questionnaire. $J$ Gerontol 1981;36:428-34.

54 Kroenke K, Spitzer RL, Williams JB. The PHQ-9: validity of a brief depression severity measure. J Gen Intern Med 2001;16:606-13.

55 Guigoz Y, Vellas B, Garry PJ. Assessing the nutritional status of the elderly: the mini nutritional assessment as part of the geriatric evaluation. Nutr Rev 2009;54:S59-65.

56 Eifert GH, Thompson RN, Zvolensky MJ, et al. The cardiac anxiety questionnaire: development and preliminary validity. Behav Res Ther 2000;38:1039-53.

57 Sciurba F, Slivka W. Six-Minute walk testing. Semin Respir Crit Care Med 1998;19:383-92.

58 Williams JB, McConnell G, Allender JE, et al. One-Year results from the first US-based enhanced recovery after cardiac surgery (ERAS cardiac) program. J Thorac Cardiovasc Surg 2019;157:1881-8.

59 Engelman DT, Ben Ali W, Williams JB, et al. Guidelines for perioperative care in cardiac surgery: enhanced recovery after surgery Society recommendations. JAMA Surg 2019;154:755-66.

60 Heart and stroke Foundation of Canada. Living well with heart disease. Toronto: heart and Stroke, 2016.

61 Pilote L, Karp I. GENESIS-PRAXY (gender and sex determinants of cardiovascular disease: from bench to beyond-Premature acute coronary syndrome). Am Heart J 2012;163:741-6.

62 Afilalo J, Lauck S, Kim DH, et al. Frailty in Older Adults Undergoing Aortic Valve Replacement: The FRAILTY-AVR Study. J Am Coll Cardiol 2017;70:689-700.

63 Wyrwich KW, Tierney WM, Babu AN, et al. A comparison of clinically important differences in health-related quality of life for patients with chronic lung disease, asthma, or heart disease. Health Serv Res 2005;40:577-92.

64 Williamson EJ, Forbes A, White IR. Variance reduction in randomised trials by inverse probability weighting using the propensity score. Stat Med 2014;33:721-37.

65 Institute for Healthcare Improvement. Plan-Do- Study-Act (PDSA) worksheet. IHI's QI Essentials Toolkit, 2017. Available: http://www.ihi. org/resources/Pages/Tools/PlanDoStudyActWorksheet.aspx

66 Canadian Institute for Health Research. Patient engagement, 2014. Available: http://www.cihr-irsc.gc.ca/e/45851.html

67 Fujiwara Y, Suzuki H, Yasunaga M, et al. Brief screening tool for mild cognitive impairment in older Japanese: validation of the Japanese version of the Montreal cognitive assessment. Geriatr Gerontol Int 2010;10:225-32. 